ARTICLE

Received 6 Nov 2013 | Accepted 30 Jan 2014 | Published 24 Feb 2014 DOI: 10.1038/ncomms4358

\title{
Phase evolution for conversion reaction electrodes in lithium-ion batteries
}

Feng Lin ${ }^{1,2}$, Dennis Nordlund ${ }^{3}$, Tsu-Chien Weng ${ }^{3}$, Ye Zhu ${ }^{4,5}$, Chunmei Ban ${ }^{6}$, Ryan M. Richards ${ }^{2}$ \& Huolin L. Xin ${ }^{7}$

The performance of battery materials is largely governed by structural and chemical evolutions during electrochemical reactions. Therefore, resolving spatially dependent reaction pathways could enlighten mechanistic understanding, and enable rational design for rechargeable battery materials. Here, we present a phase evolution panorama via spectroscopic and three-dimensional imaging at multiple states of charge for an anode material (that is, nickel oxide nanosheets) in lithium-ion batteries. We reconstruct the threedimensional lithiation/delithiation fronts and find that, in a fully electrolyte immersion environment, phase conversion can nucleate from spatially distant locations on the same slab of material. In addition, the architecture of a lithiated nickel oxide is a bent porous metallic framework. Furthermore, anode-electrolyte interphase is found to be dynamically evolving upon charging and discharging. The present study has implications for resolving the inhomogeneity of the general electrochemically driven phase transition (for example, intercalation reactions) and for the origin of inhomogeneous charge distribution in large-format battery electrodes.

\footnotetext{
${ }^{1}$ Environmental Energy Technologies Division, Lawrence Berkeley National Laboratory, Berkeley, California 94720, USA. ${ }^{2}$ Department of Chemistry and Geochemistry, Materials Science Program, Colorado School of Mines, Golden, Colorado 80401, USA. ${ }^{3}$ Stanford Synchrotron Radiation Lightsource, SLAC National Accelerator Laboratory, Menlo Park, California 94025, USA. ${ }^{4}$ Monash Centre for Electron Microscopy, Monash University, Victoria 3800, Australia. ${ }^{5}$ Department of Materials Engineering, Monash University, Victoria 3800, Australia. ${ }^{6}$ Chemical and Materials Science Center, National Renewable Energy Laboratory, Golden, Colorado 80401, USA. ${ }^{7}$ Center for Functional Nanomaterials, Brookhaven National Laboratory, Upton, New York 11973, USA.

Correspondence and requests for materials should be addressed to F.L. (email: flin@lbl.gov) or to H.L.X. (email: hxin@bnl.gov).
} 
P hase conversions are ubiquitous and fundamentally important in many aspects of materials science research including colloidal synthesis ${ }^{1}$ and lithium chemistry ${ }^{2,3}$. The response of a material to phase conversions on the nanoscale can directly dictate the performance of various energy materials in electrochemical reactions, such as fuel cell nanocatalysts ${ }^{4,5}$ and battery electrodes ${ }^{2,3,6-13}$. Specifically, phase conversion reactions have provided a rich playground for lithium-ion battery technologies with potential to improve specific/rate capacity and achieve high resistance to lithium metal plating ${ }^{14-19}$. Among the many potential candidates, transition metal oxides have received broad interests as lithium-ion battery anode materials ${ }^{20-27}$. Although electrochemistry and synthesis of transition metal oxides have been well studied ${ }^{18,19}$, the spatially resolved phase conversion pathways (for example, nucleation, charge distribution and anode-electrolyte interface (AEI) formation) remain elusive. To understand these nanoscale pathways could impart knowledge not only to unravel the underlying mechanism/challenges of conversion reactions ${ }^{28}$ but also to reveal the origin of inhomogeneous charge distribution in large-format battery electrodes ${ }^{10,29,30}$.

In recent years, in situ transmission electron microscopy (TEM) studies have been performed to investigate the electrochemically driven phase conversions in lithium-ion batteries. However, most of these studies primarily focused on non-volatile environments and lacked information on interfaces between commercially used organic liquid electrolytes and battery electrodes $^{8,11}$. Furthermore, due to limitations of TEM sample geometry in these studies, electrode-electrolyte interfaces were simplified as a point/line contact and thus failed to represent the electrode materials' three-dimensional (3D) immersion in electrolyte that occurs in practical battery cells. Other groups have attempted quasi in situ observation of energy materials supported on TEM grids in liquid electrochemical cells ${ }^{31}$. However, due to the limited loading allowed on a TEM grid, an accurate and

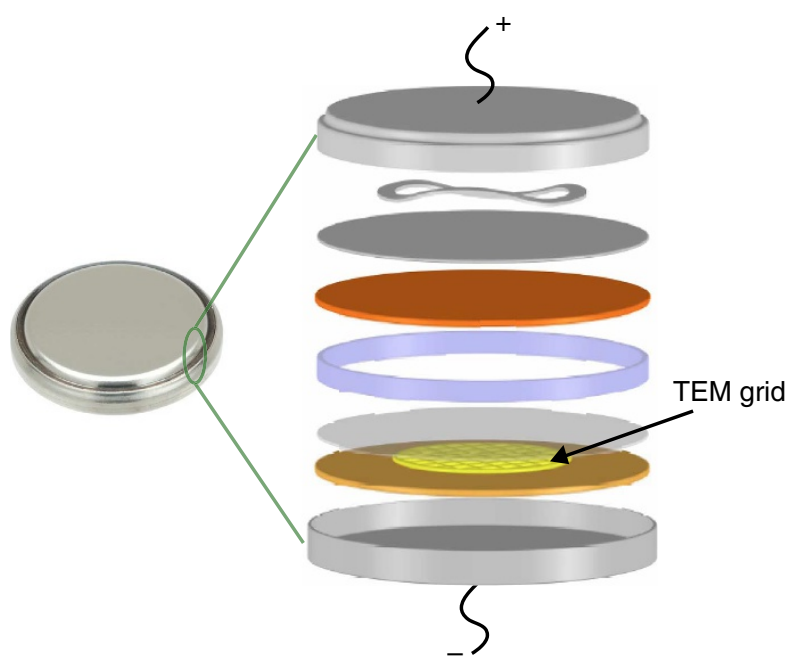

Figure 1 | Schematic illustration of a coin cell design loaded with a TEM grid. In the coin cell, a holey carbon supported TEM grid, which was loaded with a small amount of the anode material, was pressed against the bulk anode to ensure the materials on the grid are submerged in the same electrolyte environment as the bulk anode. From top to bottom: anode cap, spring, spacer, lithium anode, gasket, separator, TEM grid loaded with $\mathrm{NiO}$ nanosheets, working electrode and cathode cap. A NiO-based working electrode is considered as a 'cathode' in this half-cell configuration but as an 'anode' in the full-cell configuration. Therefore, a NiO-based working electrode is identified as an 'anode' in this paper. reproducible charging/discharging rate (C-rate) control is difficult to achieve but is essential for lithium-ion battery systems. Here, we report a direct visualization of phase conversion in a high performance anode material in a coin cell configuration. In the coin cell, we pressed a holey carbon supported TEM grid, which was loaded with a small amount of the anode material, against the bulk anode to ensure that the materials on the grid are submerged in the same electrolyte environment as the bulk anode (Fig. 1). This configuration allows us to accurately control the C-rate for cycling; in addition, the TEM grid can be dissembled from the cell to facilitate TEM imaging and spectroscopy. This grid-in-a-coin cell methodology has enabled direct probing of the $3 \mathrm{D}$ morphology and charge state distribution of the anode material and evolution of AEI at different stages of electrochemical cycling with atomic-scale TEM imaging, electron tomography, electron energy loss spectroscopic mapping as well as ensemble-averaged synchrotron X-ray spectroscopy.

Our charge mapping and $3 \mathrm{D}$ reconstructions of the electrochemically cycled materials reveal that during both lithiation and delithiation, phase conversions can initiate and propagate from spatially distant locations on the same slab of anode material. This suggests a heterogeneous phase conversion mechanism, analogous to heterogeneous nucleation in crystal growth, can be dominant and fundamentally important in the conversion reaction. The present study captures previously unexpected heterogeneous nucleation characteristics in electrochemically driven phase conversions in metal oxides, providing insights into designing practically viable conversion reaction electrodes in general.

\section{Results}

Morphology and electronic structure of $\mathrm{NiO}$ nanosheets. Owing to its high theoretical capacity, $\mathrm{NiO}$ and its nanocomposites have been extensively studied as potential anodes for highcapacity lithium-ion batteries ${ }^{18,19}$. Here, structurally well-defined $\mathrm{NiO}$ material was prepared using a solvothermal method aided with a pseudo-supercritical drying technique ${ }^{32}$. The as-prepared $\mathrm{NiO}$ exhibits a sheet-like structure with hexagonal holes (Fig. 2a,b) and a thickness of $24 \pm 8 \mathrm{~nm}$ (Supplementary Fig. 1). The atomic resolution annular dark-field scanning TEM (ADFSTEM) image reveals that nickel ions are packed into a hexagonal arrangement with the zone axis aligned perpendicular to the nanosheet (Fig. 2c) indicating that the surface primarily consists of rock salt (111) facets. The formal oxidation state of nickel is $2+$ in $\mathrm{NiO}$ nanosheets as determined by soft X-ray absorption spectroscopy (XAS) and spatially resolved electron energy loss spectroscopy (EELS), both of which correspond to the dipole allowed transitions from Ni $2 \mathrm{p}^{6} 3 \mathrm{~d}^{8}$ to $\mathrm{Ni} 2 \mathrm{p}^{5} 3 \mathrm{~d}^{9}$ states, including $\mathrm{Ni} 2 \mathrm{p}^{3 / 2}$ and Ni $2 \mathrm{p}^{1 / 2}$ spin-orbit final states ${ }^{33,34}$ (Fig. $2 \mathrm{~d}, \mathrm{e}$ ). The pre-edge feature in the $\mathrm{O} \mathrm{K}$-edge corresponds to the $\mathrm{p}$-character of the Ni3d-O2p hybridized states in the $\mathrm{NiO}_{6}$ octahedral unit ${ }^{35}$. The $\mathrm{O}$ K-edge feature associated with $\mathrm{Ni3d}-\mathrm{O} 2 \mathrm{p}$ hybridization states in the pristine $\mathrm{NiO}$ nanosheet is equivalently observed using XAS and EELS (Fig. 2d,e). The consistency between XAS and EELS suggests the homogeneity of the pristine material and demonstrates the capability of performing correlated studies for efficient phase identification and charge mapping.

Electrochemical performance of $\mathrm{NiO}$ nanosheets. Chargedischarge voltage profile shows distinct phase conversion plateaus for the $\mathrm{NiO} / \mathrm{Ni}$ redox couple (Fig. 3a). The $\mathrm{NiO}$ reduction potentials are $0.6 \mathrm{~V}$ and $1.0-1.5 \mathrm{~V}$ for the first cycle and subsequent cycles, respectively. The charge-discharge redox behaviour remained intact when a TEM grid was included in the coin cell (Supplementary Fig. 2). Supplementary Figure 3 shows 

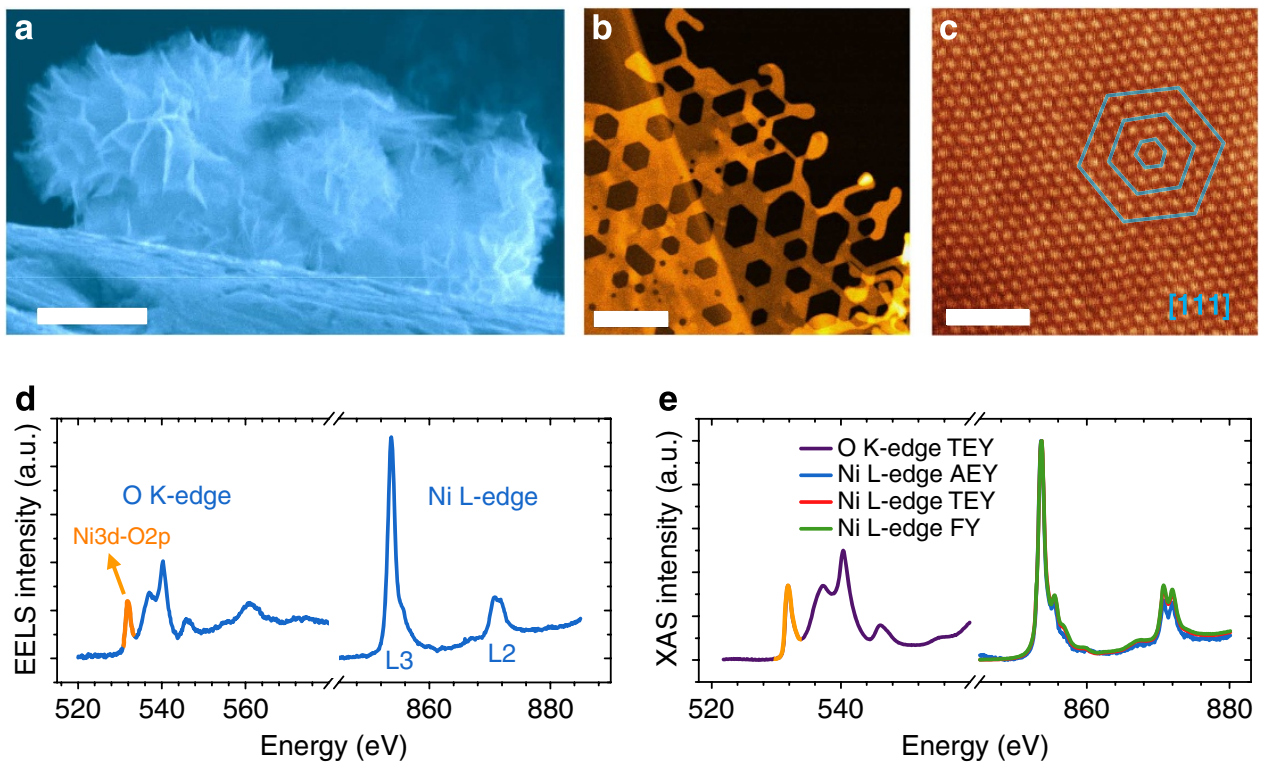

Figure 2 | Pristine NiO nanosheets. (a) SEM image of NiO nanosheet bundles supported on a stainless steel substrate. (b) ADF-STEM overview image of an individual $\mathrm{NiO}$ nanosheet. (c) ADF-STEM image of a NiO nanosheet along the [111] zone axis. The hexagons are drawn for the visual enhancement of the hexagonal arrangement of nickel ion columns. (d) EELS O K-edge and Ni L-edge spectra for a NiO nanosheet ( $\sim 0.8 \mathrm{eV}$ energy resolution). (e) XAS O K-edge and Ni L-edge spectra for a pristine NiO electrode, where the XAS O K-edge spectrum was collected using TEY mode and XAS Ni L-edge spectra were collected using AEY, TEY and FY modes. XAS Ni L-edge spectra show the bulk and surface consistency of the pristine $\mathrm{NiO}$ electrode. The scale bars are $5 \mu \mathrm{m}, 100 \mathrm{~nm}$ and $1 \mathrm{~nm}$ in (a), (b) and (c), respectively.

the cyclic voltammetry for the first two cycles, where the voltage ranges corresponding to the $\mathrm{NiO}$ reduction potentials are identified. At $\mathrm{C} / 2$, the first discharge and charge capacities are $1,138 \mathrm{mAh}^{-1}$ and $808 \mathrm{mAg}^{-1}$, respectively. The first cycle coulombic inefficiency is attributed to the incomplete decomposition of AEI and other factors including kinetic limitations in the charging process ${ }^{26}$. The charge-discharge voltage hysteresis showed negligible change at different $\mathrm{C}$ rates (Fig. 3a), which suggests that the reaction pathway remained intact ${ }^{18}$. Coulombic efficiency is improved to $98 \%$ for the second cycle at $\mathrm{C} / 2$ and shows little degradation with extended charge-discharge cycles (Fig. 3b). Specific charge capacity reaches a peak of $928 \mathrm{mAh} \mathrm{g}^{-1}$ and remains remarkably high $\left(675 \mathrm{mAh} \mathrm{g}^{-1}\right)$ after 40 cycles (Fig. 3b). The higher capacity relative to the theoretical capacity $\left(718 \mathrm{mAh} \mathrm{g}^{-1}\right)$ suggests that interfacial charge storage and/or reversible side reactions play roles in the extra capacity ${ }^{22,36}$. The reversible side reactions probably include but are not limited to lithium storage in carbon black ${ }^{37}$ and $\mathrm{CuO}^{38}$ at the surface of the $\mathrm{Cu}$ current collector, and reversible conversion of $\mathrm{LiOH}$ to $\mathrm{Li}_{2} \mathrm{O}$ and $\mathrm{LiH}^{39}$. An extraordinary high-rate capability is demonstrated in Fig. $3 c$ in which the cell retains a specific capacity of $755 \mathrm{mAh} \mathrm{g}^{-1}$ at $5 \mathrm{C}$ and is fully recovered at C/2 after a series of high-rate charge-discharge cycles. Impedance Nyquist plots were collected after high-rate cycling and are shown in Fig. 3d. The Nyquist spectra are composed of semicircles at high frequency and Warburg tails at low frequency. The semicircles include both charge transfer resistance and thin film resistance. Detailed analysis of the semicircles is provided in Supplementary Fig. 4 and Supplementary Table 1. Briefly, the thin film resistance was gradually increased after charge-discharge cycles due to the AEI formation. However, the charge transfer resistance underwent significant reduction after charge-discharge cycles, especially after high-rate cycling (Fig. 3d, Supplementary Fig. 4 and Supplementary Table 1 ), which suggests that the superior rate capability is probably attributed to the improved charge transfer. Overall, the electrochemical performance of $\mathrm{NiO}$ nanosheets is superior to the reported $\mathrm{NiO}$ anode materials in the literature ${ }^{25,40}$, which renders $\mathrm{NiO}$ nanosheets an excellent candidate to study phase change in conversion reactions. Furthermore, $\mathrm{NiO}$ is a member of the conversion reaction family and its behaviour is expected to be transferrable to other high performance $3 \mathrm{~d}$ transition metal oxide anodes such as $\mathrm{Fe}_{2} \mathrm{O}_{3}, \mathrm{CoO}$ and $\mathrm{MnO}_{2}$.

Surface chemistry and phase conversion in $\mathrm{NiO}$ nanosheets. To begin with, we achieved ensemble-averaged characteristics for composite electrodes under various states of charge (SOC). The intensity of XAS in the Auger electron yield (AEY) mode is sensitive to the overlayer thickness $(d)$ at the surface of a material due to the limited mean free path of Auger electrons in the overlayer. Under identical Auger electron signal collection configurations, the AEY intensity is empirically in an exponential relationship with $(-d)$. Therefore, any chemical build-up at the surface of $\mathrm{NiO}$ nanosheets is evidenced by the decreased intensity of XAS in the AEY mode. Here, we observe that the Ni L-edge AEY intensity is dynamically altered depending on the SOC. As shown in Fig. 4a, the AEY signal was completely quenched in the fully discharged electrode and partially recovered in the charged state. This observation suggests that electrochemical processes govern the thickness of the surface overlayer, that is, the surface overlayer was developed and decomposed during discharging and charging, respectively. The normalized Ni L-edge spectra (Supplementary Fig. 5) clearly show the changes of nickel oxidation states corresponding to the phase conversion plateaus in the charge-discharge voltage profiles (Fig. 3a). A comparison of XAS O K-edge spectra for the pristine and cycled electrodes provides direct evidence for chemical evolution in a large scale. As shown in Fig. 4b, the characteristic O K-edge peak associated with the Ni3d-O2p hybridization state is significantly degraded. In contrast, the intensity related to $\pi^{*}(\mathrm{C}=\mathrm{O})^{41}$ experiences dramatic enhancement in the cycled electrode (red spectrum in Fig. $4 \mathrm{~b})$. The $\pi^{\star}(\mathrm{C}=\mathrm{O})$ is likely attributed to carbonate groups at the surface of nanosheets (see discussion below). The overlayer on 


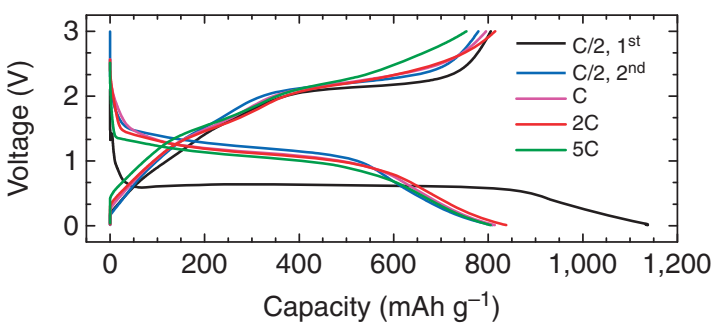

b
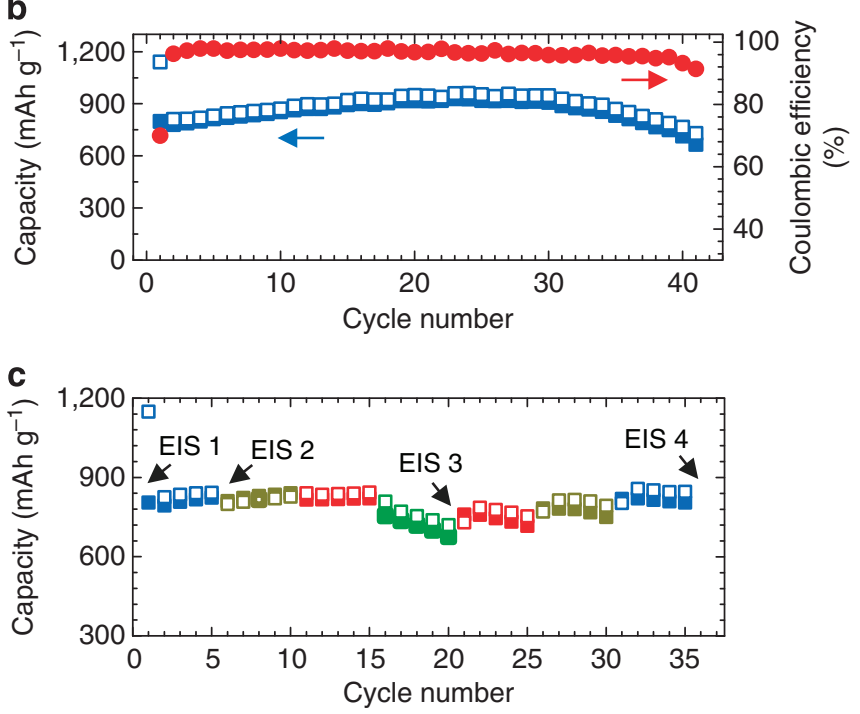

d

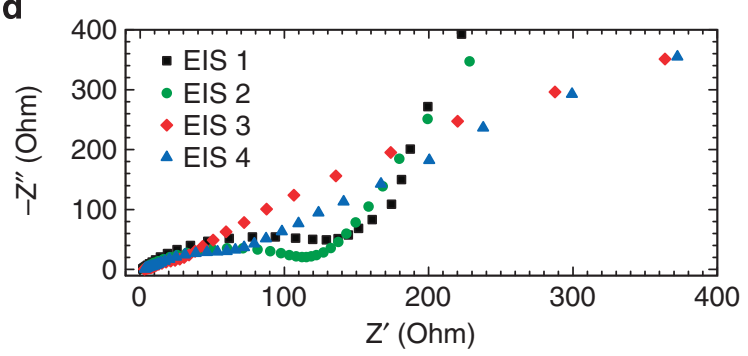

Figure 3 | Battery cycling performance of NiO nanosheets.

(a) Charge-discharge voltage profiles at different $C$ rates.

(b) Capacity retention (left $\mathrm{y}$-axis) and coulombic efficiency (right $\mathrm{y}$-axis) at C/2 rate. (c) Rate capability: C/2 (blue), C (yellow), 2 C (red) and $5 \mathrm{C}$ (green). (d) Electrochemical impedance Nyquist plots after charge-discharge cycles and the corresponding EIS collection points are indicated in (c). The charge capacity and discharge capacity are indicated, respectively, by solid and open cubes in (b) and (c).

nanosheets was visually confirmed by STEM imaging, and it is found that the overlayer occupies the surfaces of both $\mathrm{NiO}$ and $\mathrm{Ni}$ patches. A vast number of EELS spectra were acquired in the lowdose mode for the overlayer (that is, AEI) and the representative spectra are shown in Fig. 4d. The existence of carbonate groups is further confirmed with EELS C K-edge, where the sharp peak at $292.5 \mathrm{eV}$ and the broad peak centred at $302.8 \mathrm{eV}$ are attributed to the unoccupied $\pi^{*}$ and $\sigma^{*}$ antibonding molecular orbitals of $\mathrm{CO}_{3}{ }^{2-}$ clusters $^{42}$. (Careful alignment of EELS energy axis was performed by cross aligning the $\mathrm{C}$ K-edge of the nearby amorphous carbon with the standard spectrum.) The major feature of EELS O K-edge is in accordance with that observed in XAS O K-edge. Furthermore, the EELS Li K edge spectrum is in agreement with that reported for $\mathrm{Li}_{2} \mathrm{CO}_{3}$ (ref. 43). In addition, localized $\mathrm{Li}_{2} \mathrm{CO}_{3}$ nanocrystals were observed using high-resolution TEM imaging and fast Fourier transform (Supplementary Fig. 6).
Finally, one may conclude that the AEI likely consists of $\mathrm{Li}_{2} \mathrm{CO}_{3}$ domains in a complex amorphous organic matrix, which is similar to those reported for graphite anode materials ${ }^{4-47}$.

Next, phase conversion process was studied and previously unknown characteristics were revealed for conversion reactions. Figure 5a-e show the representative STEM images for $\mathrm{NiO}$ nanosheets with various SOC. Electrochemically driven phase conversion has been interpreted as a progressive nucleation/ propagation from the surface of a particle until the particle is fully converted, that is, core/shell model or homogeneous phase conversion ${ }^{48}$. In reality, conditions for homogenous phase conversion are hardly satisfied on the nanoscale, and any fluctuations on the electrolyte exposure, electrical contact or crystal defects will foster heterogeneous phase conversion. Our observation reveals that heterogeneous phase conversion can be dominant even in ultrathin nanosheets. Figure $5 b$,d demonstrate heterogeneous phase conversion phenomena during lithiation and delithiation processes, respectively. During lithiation, nanoscale nickel networks preferentially nucleate in a number of spatially distant locations on a single $\mathrm{NiO}$ nanosheet and then propagate into the vicinity until the propagation fronts meet, as indicated by the arrows in Fig. 5b. The opposite reaction, $\mathrm{NiO}$ formation, exhibits nearly identical heterogeneous characteristics, as shown in Fig. 5d. Supplementary Figure 7 provides more S/TEM images for the heterogeneous phase conversion phenomena. After a full cycle, the majority of $\mathrm{NiO}$ nanosheets were retained in the crystalline structure, as shown in Fig. 5e. The lattice resolution image of the delithiated $\mathrm{NiO}$ material and the bending contours in the low-magnification TEM image (Supplementary Fig. 8) clearly demonstrate that the cycled $\mathrm{NiO}$ remains crystalline with a crystalline domain size of $\sim 100 \mathrm{~nm}$. Charge distribution mapping was performed on a region with heterogeneous phase conversion using $\mathrm{Ni}$ L-edge EELS spectroscopy, as shown in Fig. 5f-i. The mapping provides a direct visualization for the lithiation process. The area coloured in green represents the $\mathrm{NiO}$ that has already been converted to $\mathrm{Ni}$ metal, while no phase conversion has occurred in the area coloured in red. EELS line scans were performed at the vicinity of a phase propagation front, as shown in Fig. 5j. Scanning from the pure $\mathrm{Ni}$ region to the pure $\mathrm{NiO}$ region (Fig. 5j), we observed a gradual evolution in the shapes and intensities of $\mathrm{Ni} \mathrm{L}_{2,3}$ nearedge fine structures due to the variation of nickel oxidation states (Fig. 5k). The spectra with mixed nickel oxidation states could be decomposed using a linear combination of $\mathrm{Ni}^{0}$ and $\mathrm{Ni}^{2+}$ fingerprints, $\mathrm{Ni}^{0}$ and $\mathrm{Ni}^{2+}$ profiles are plotted as a function of the relative position along the scanning line (Fig. 5j). In general, $\mathrm{Ni}^{0}$ and $\mathrm{Ni}^{2}+$ profiles exhibit opposite trends along the scanning direction. A mixed-state region was observed at the boundary between the pure $\mathrm{Ni}^{0}$ region and the pure $\mathrm{Ni}^{2+}$ region (Fig. 51 ). This asymmetric distribution of nickel valence states suggests a unidirectional propagation of the phase conversion front, which is a characteristic of heterogeneous phase conversion.

The visualization of a heterogeneous phase conversion was achieved by reconstructing the $3 \mathrm{D}$ internal nanostructures for a nanosheet with $50 \%$ SOC (during the charging process) by ADF-STEM tomography. Figure 6a shows the snapshots for the reconstructed tomography on different orientations, and the associated full-view movie is provided in Supplementary Movie 1. Nickel nanoparticles are discernible in the $\mathrm{NiO}$ matrix close to the propagation fronts that are indicated by the green arrows in Fig. 6a. These imbedded nickel nanoparticles would otherwise be ignored in projection images but validate the mixed nickel oxidation state at the boundary between the pure $\mathrm{Ni}^{0}$ region and the pure $\mathrm{Ni}^{2+}$ region (Fig. 5l). Furthermore, we noticed the morphology of the $\mathrm{Ni}^{0}$ region was different from the conventional understanding ${ }^{20,24,49-51}$. With projection images, it is 


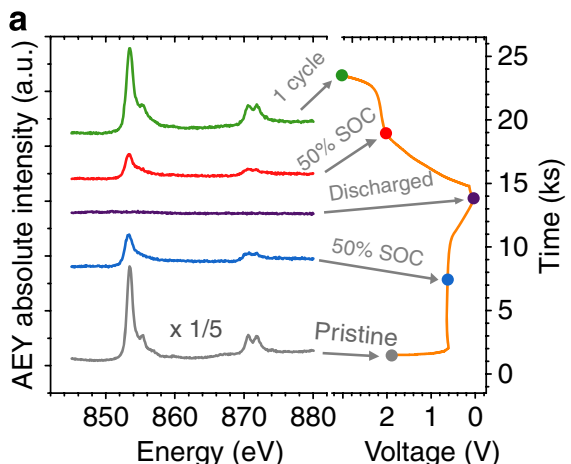

C

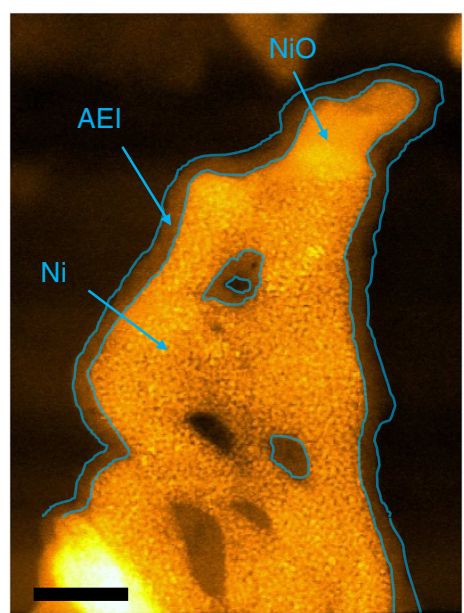

b
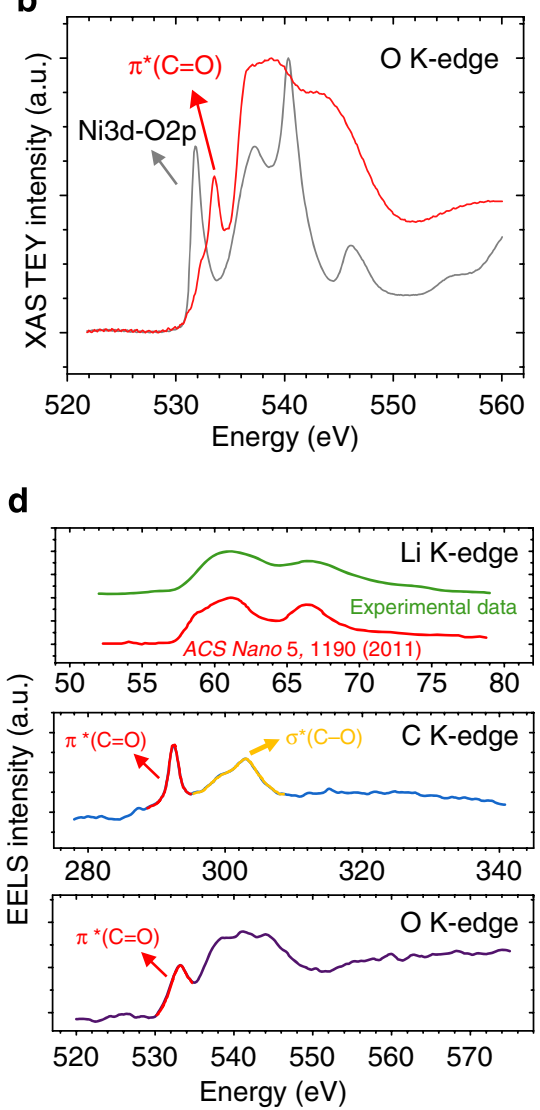

Figure 4 | Chemical evolution at the surface of NiO nanosheets. (a) XAS/AEY spectra for electrodes at different SOC, and the voltage-time profile is provided in parallel to indicate the states where data collection was accomplished. XAS/AEY is a surface detection mode and the Ni L-edge signal disappears at the fully discharged state due to a thick coating of AEI on the surface. (b) XAS/TEY O K-edge spectra for electrodes in the pristine state (grey) and in the $50 \%$ SOC (red), and the assignment of peaks is indicated in the figure. (c) STEM image of a NiO nanosheet during a discharging process, and $\mathrm{NiO}, \mathrm{Ni}$ and $\mathrm{AEl}$ are indicated with arrows in the image. The scale bar is $50 \mathrm{~nm}$. (d) EELS Li K-edge, C K-edge and O K-edge spectra for the AEl layer, and the assignment of peaks is indicated in the figure. A reference $\mathrm{Li} \mathrm{K}$-edge spectrum of $\mathrm{Li}_{2} \mathrm{CO}_{3}$ (ref. 43) is provided in the figure.

possible to incorrectly perceive that individual discrete $\mathrm{Ni}$ nanoparticles are imbedded in the complex compounds containing $\mathrm{Li}, \mathrm{O}$ and $\mathrm{C}^{20,24,49,50}$. However, projection images could be misleading in terms of distinguishing chemically interconnected nanoparticles from physically overlapped nanoparticles. True morphology can only be visualized by $3 \mathrm{D}$ tomography. In the lithiated region of Fig. 6a, nickel nanoparticles are interconnected into a porous network and exhibit greatly distinct characteristics in contrast to the widely accepted model of discrete spherical metal nanoparticles ${ }^{20,24,49,50}$. The interconnected nickel networks are further supported by the high-magnification reconstructed tomography of the lithiated region, as shown in Supplementary Movie 2. The heterogeneous nucleation phenomenon is also dominant during the discharging process, and the reconstructed images are shown in Supplementary Fig. 9 and Supplementary Movie 3. The porous metallic structure is completely retained towards the end of a discharging process, as shown in Fig. 6b. Supplementary Movie 4 illustrates the full-view panorama for the interconnecting framework. Finally, the benefit of the 3D metallic framework not only lies in the improved electron transfer but also the structural integrity (that is, inhibit pulverization) of $\mathrm{NiO}$ nanosheets thus allowing for durable high-rate cycling. This is direct evidence of a nanoporous metallic framework in conversion reactions. The result suggests that enhanced durability could be realized for conversion reaction electrodes if appropriate cycling conditions are applied to exclusively yield nanoporous frameworks. It is noted that pore size in the framework is much smaller than electrochemically dealloyed metallic porous structures in the literature ${ }^{52}$, which enables potential applications of the present porous frameworks in catalysis and sensing. Another unexpected phenomenon is the bending of nanosheets after a complete lithiation step (Fig. $6 \mathrm{~b}$ and Supplementary Movie 4), which is rarely observed after a complete delithiation step. We infer that the bending phenomenon is largely attributed to the electrochemically induced plastic deformation of the metallic porous framework. Presumably, the energy associated with the plastically deformed bending originates from the electrical energy supplied by the external circuit during the discharging process. Therefore, the reversibility of such bending under extended charge-discharge cycles should impose effects on the efficiency of battery cycling.

Mechanism of heterogeneous nucleation in $\mathrm{NiO}$ nanosheets. The origin of heterogeneous phase conversion could be attributed to inhomogeneous electrolyte exposure, electrical contact and/or intrinsic crystal defects, such as grain boundaries. It is unlikely the origin of heterogeneous nucleation at the atomic scale will be elucidated using X-ray based imaging techniques ${ }^{48,53}$. Specifically, we observed that $\mathrm{Ni}$ nucleation preferentially emerges at grain boundaries, as shown in Fig. 7a. The orientations of the two 

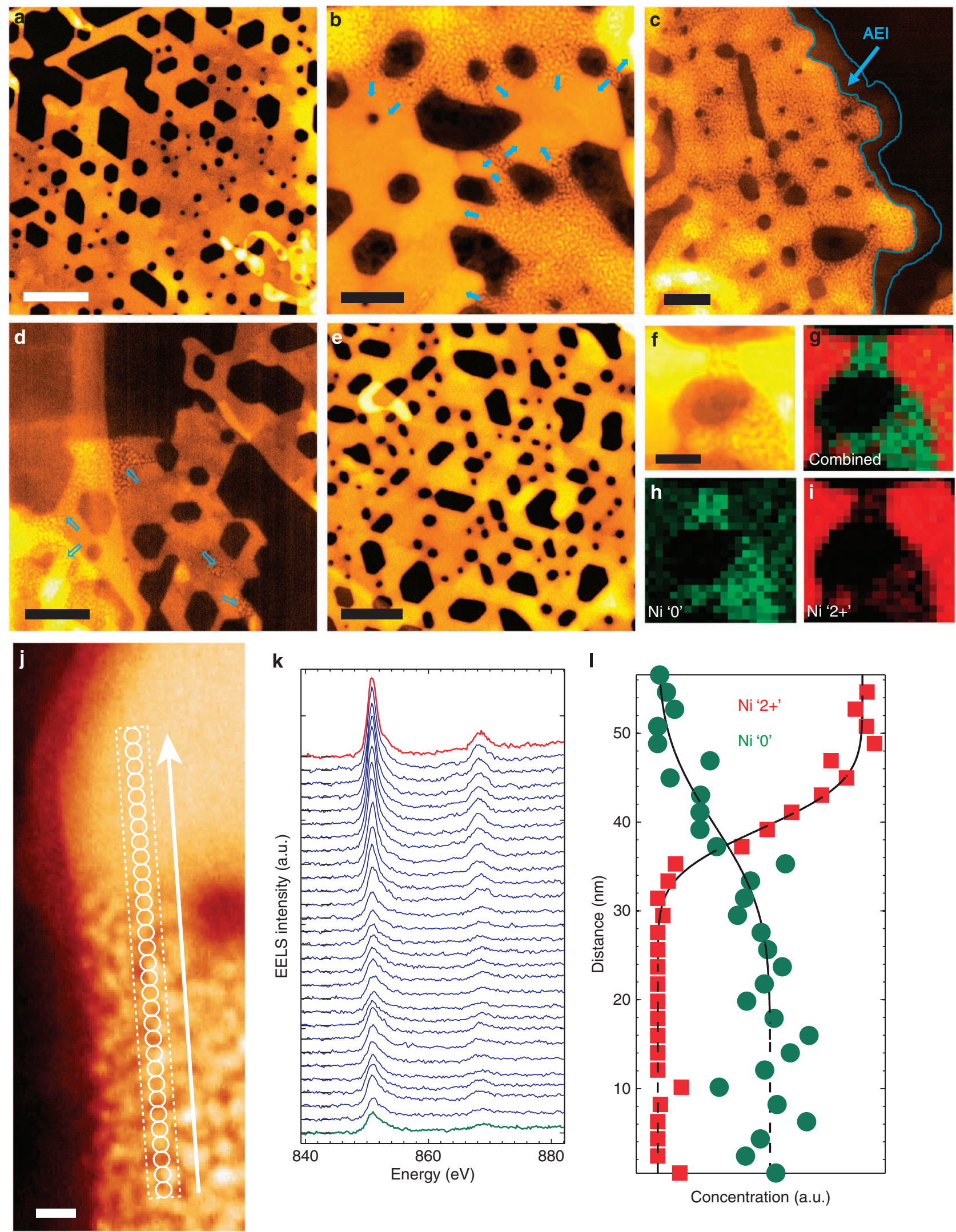

Figure 5 | STEM imaging and charge mapping. (a) Pristine $\mathrm{NiO}$ nanosheet. (b) NiO nanosheet with a $50 \%$ SOC (discharging). (c) NiO nanosheet with a fully discharged state. (d) NiO nanosheet with a $50 \%$ SOC (charging). (e) NiO nanosheet with a full cycle. (f-i) Charge mapping for a localized region with a 50\% SOC, where (f), (g), (h) and (i) are STEM image, combined mapping profile, Ni ' $\mathrm{O}^{\prime}$ mapping (green) and $\mathrm{Ni}$ ' $2{ }^{+}$' mapping (red), respectively. (j-I) EELS line profile, where $(\mathbf{j}),(\mathbf{k})$ and $(\mathbf{I})$ are STEM image, EELS spectra and $\mathrm{Ni}$ ' $\mathrm{O}$ ' $/ \mathrm{Ni}$ ' $2+{ }^{\prime}$ concentration profiles, respectively. The arrows in (b) and (d) indicate the propagation direction of NiO-to-Ni and Ni-to-NiO conversions, respectively. The scale bars are $100 \mathrm{~nm}, 50 \mathrm{~nm}$, $50 \mathrm{~nm}, 50 \mathrm{~nm}, 100 \mathrm{~nm}, 20 \mathrm{~nm}$ and $5 \mathrm{~nm}$ in (a), (b), (c), (d), (e), (f) and (j), respectively. 
a

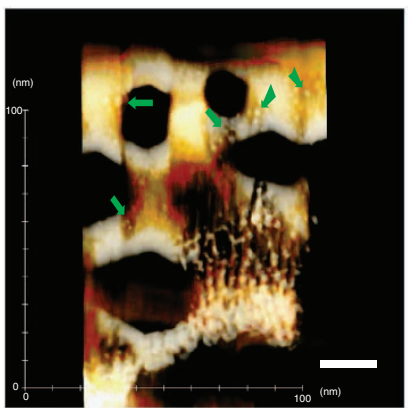

b
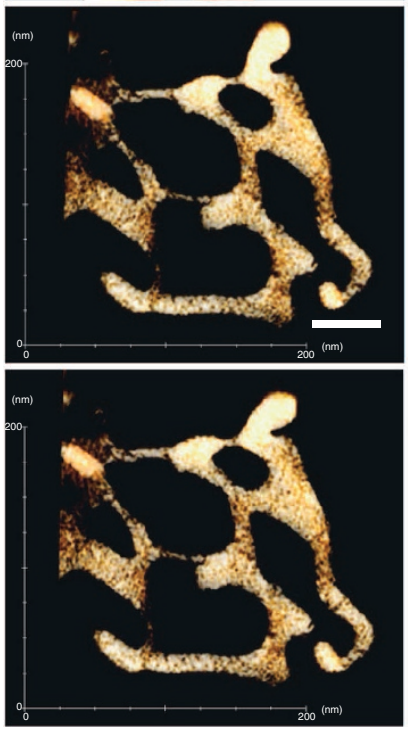
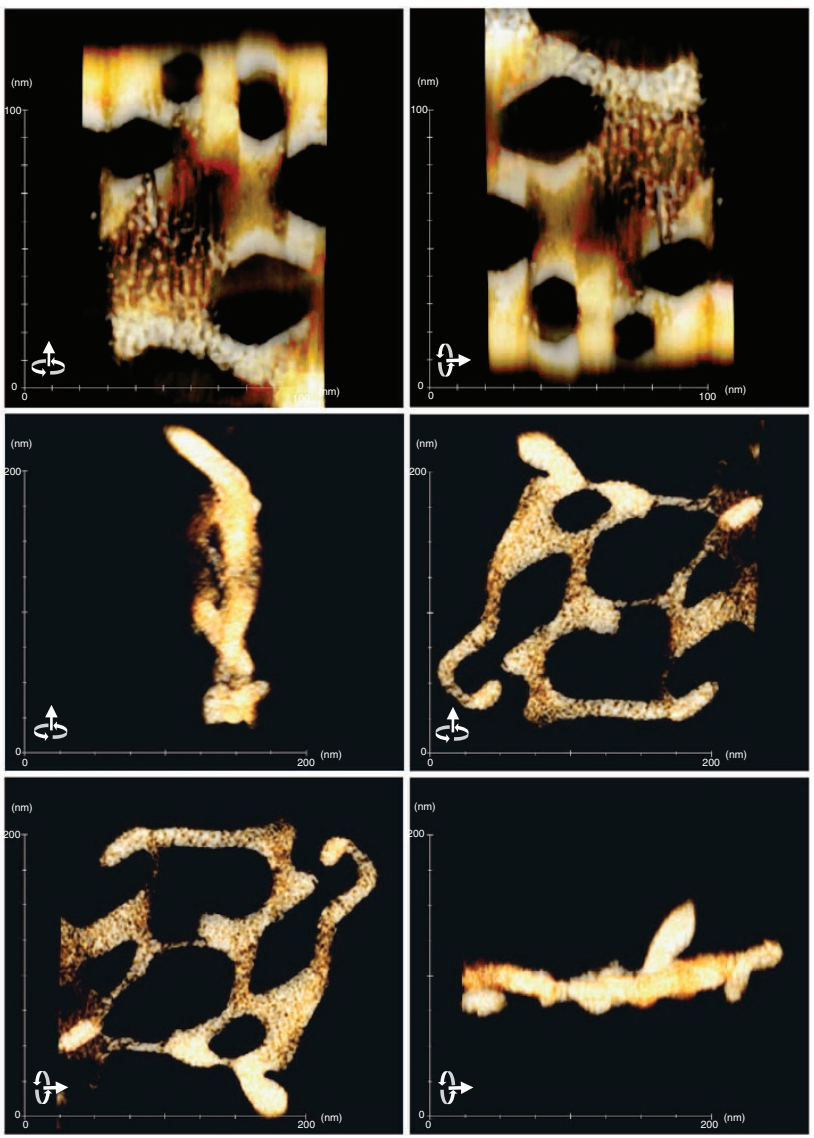

Figure 6 | 3D STEM tomography. (a) A reconstructed $\mathrm{NiO}$ nanosheet with $50 \%$ SOC during charging process, and the green arrows exemplify the $\mathrm{Ni}$ nanoparticles that are imbedded in the $\mathrm{NiO}$ matrix as a result of heterogeneous nucleation. (b) A reconstructed $\mathrm{NiO}$ nanosheet in the fully discharged state. The electrochemically reduced $\mathrm{Ni}$ forms a bent porous metallic framework. The rotation operations are indicated in the figures. See Supplementary Information for complete movies (Movies 1 and 4). The scale bars are $20 \mathrm{~nm}$ and $50 \mathrm{~nm}$ in (a) and (b), respectively.

a

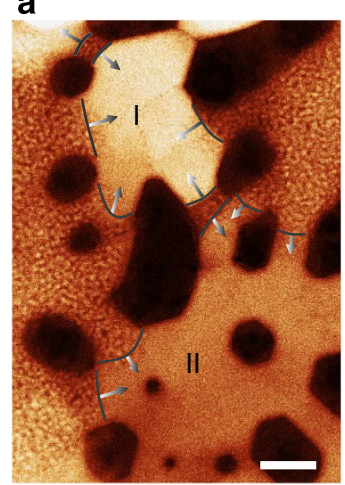

b

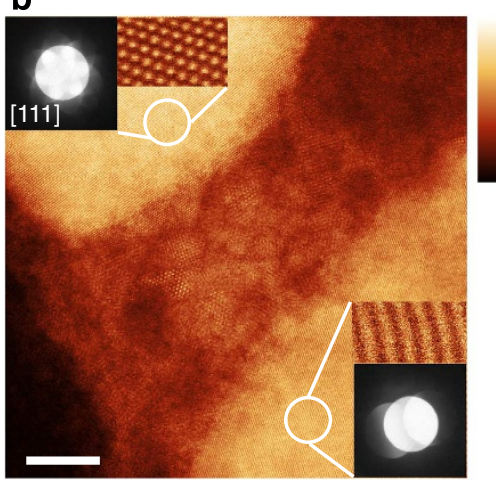

Figure 7 | Propagation of heterogeneous nucleation at grain boundaries. (a) Low-magnification high-angle ADF-STEM (HAADF-STEM) image showing heterogeneous nucleation with multiple nucleation fronts indicated by bending contours and arrows. (b) Atomic resolution HAADF-STEM image for a selected phase conversion scene, where the insets show atomic arrangements and convergent beam electron diffraction patterns for $\mathrm{NiO}$ patch I and $\mathrm{NiO}$ patch II in (a). The scale bars are $30 \mathrm{~nm}$ and $5 \mathrm{~nm}$ in (a) and (b), respectively.

patches of $\mathrm{NiO}$ are not registered, as demonstrated by the channelling contrast (Fig. 7a), convergent beam electron diffraction patterns (insets of Fig. 7b), and atomically resolved HAADF-STEM images (insets of Fig. 7b), indicating that there

was likely a grain boundary present between the two patches before lithiation. This observation suggests that electrochemically driven phase conversion follows the fundamental rules of phase transformation in solids that nucleation activation energy is lower at grain boundaries due to the extra energy associated with lattice distortion and dislocations ${ }^{54}$. In addition, the loosely packed lattices along grain boundaries create a rapid diffusion channel for lithium ions therefore enabling the nucleation of nickel metal nanoparticles.

\section{Discussion}

The elucidation of electrochemically driven phase conversion is fundamentally significant to interpret electrode behaviours for rechargeable batteries. On account of the unique two-dimensional morphology of $\mathrm{NiO}$ nanosheets, we were able to resolve phase conversion characteristics in a battery electrode using both spatially resolved and ensemble-averaged diagnostic tools. Our finding probes the nanoscale phase conversion and chemical evolution phenomena that are directly associated with the battery performance in practice. Firstly, the chemical environment of AEI at the surface of $\mathrm{NiO}$ nanosheets is determined to be $\mathrm{Li}_{2} \mathrm{CO}_{3}$ nanoscale domains imbedded in an amorphous organic matrix and its thickness exhibits dependence on the SOC. The chemical environment of AEI on $\mathrm{NiO}$ nanosheets is similar to that at the surface of graphite anode materials ${ }^{44,46,47}$. Therefore, these observations represent a step towards leveraging AEI on transition metal oxide materials with the conventional concept of solid-electrolyte interphase on graphite materials ${ }^{28,45}$. 
Secondly, the electrochemically produced nickel nanoparticles are constructed into a bent nanoporous metallic framework that inhibits pulverization and maintains the integrity of $\mathrm{NiO}$ nanosheets to allow extended charge-discharge cycles. This result resolves the previous misconception about the morphology of transition metal nanostructures that were exclusively identified as discrete nanoparticles $20,24,49,50$, amorphous domains ${ }^{51}$ or localized interconnected nanoparticles ${ }^{7}$. Furthermore, the nanoporous framework creates an electron diffusion network to enable high-rate capability for conversion reactions ${ }^{55}$. Thirdly, heterogeneous phase conversion was found to prevail in conversion reactions, which is likely caused in part by the preferential nucleation at grain boundaries. Presumably, the particles with different densities of grain boundaries undergo phase conversion at different rates resulting in inhomogeneous charge distribution in large-format batteries. Previous in situ TEM studies observed unidirectional phase propagation phenomena using a single point/line contact ${ }^{8}$. Our results show that the unidirectional phase propagation mechanism is still valid to some degree under more relevant battery conditions, for example, the present coin cell configuration. However, in particles with 3D electrolyte immersion and electrical contact, multiple propagation fronts proceed simultaneously in each individual nanosheet, and the phase propagation event is completed when the propagation fronts merge. This phenomenon has been overlooked in the imaging techniques that lack atomic resolution (for example, synchrotron X-ray imaging). Finally, the discrepancy between our study and a recently reported coreshell model ${ }^{48}$ is attributed to the different spatial resolution achieved in these studies. The atomic-scale investigation in the present study enriches the mechanistic paradigm for phase conversion phenomena at a complementary length scale. In summary, on the basis of the high performance $\mathrm{NiO}$ nanosheets, the present study delivers completely new insights into phase conversion and chemical evolution of transition metal oxides in atomic scale and sheds light on the inhomogeneous charge distribution in large-format battery electrodes.

\footnotetext{
Methods

Electrode preparation and battery testing. The active material ( $\mathrm{NiO}$ nanosheets) was synthesized using a solvothermal method aided with an alcohol pseudosupercritical drying technique. $\mathrm{Ni}\left(\mathrm{NO}_{3}\right)_{2} \cdot 6 \mathrm{H}_{2} \mathrm{O}$, urea and benzyl alcohol was added into $50 \mathrm{ml}$ of methanol with a molar ratio of 2:1:4. The solution was stirred for $1 \mathrm{~h}$ and transferred to an autoclave (Parr Reactor, model \#4520). The reaction mixture was purged with Ar for $1 \mathrm{~min}$ and then Ar was filled with a pressure of 9 bars before initiating the heating process. The mixture was heated to $265^{\circ} \mathrm{C}$ and maintained for $1.5 \mathrm{~h}$. Finally, the vapour inside was vented (that is, pseudosupercritical drying process). A green powder was collected and was subsequently calcined at $500^{\circ} \mathrm{C}$ for $6 \mathrm{~h}$ to yield $\mathrm{NiO}$ nanosheets. Composite electrodes were prepared with $80 \mathrm{wt} \%$ active material, $10 \mathrm{wt} \%$ polyvinylidene fluoride and $10 \mathrm{wt} \%$ acetylene carbon black in $\mathrm{N}$-methyl-2-pyrrolidone and cast onto copper current collectors with loadings of $1-2 \mathrm{mg} \mathrm{cm}^{-2}$. The packing density was calculated to be $0.7 \mathrm{~g} \mathrm{~cm}^{-3}$ using the areal loading divided by the cross-sectional thickness of the electrode (Supplementary Fig. 10). 2032-type coin cells were assembled in an argon-filled glove box using the composite electrode as the positive electrode and $\mathrm{Li}$ metal as the negative electrode. A Celgard separator 2400 and $1 \mathrm{M} \mathrm{LiPF}_{6}$ electrolyte solution in 1:1 w/w ethylene carbonate/diethyl carbonate were used to fabricate coin cells. Battery testing was performed on computer controlled VMP3 channels (BioLogic). 1C was defined as full discharging $\mathrm{NiO}$ in $1 \mathrm{~h}$, which corresponds to a specific current density of $718 \mathrm{~mA} \mathrm{~g}^{-1}$ ( $\mathrm{NiO}$ has a theoretical capacity of $718 \mathrm{mAh}^{-1}$ ). The charging current was set identical to that of the discharging in the present study. Electrochemical impedance spectra were collected after full cycles with a $10 \mathrm{mV}$ AC signal ranging from $10 \mathrm{mHz}$ to $100 \mathrm{kHz}$. Cyclic voltammetry was performed with a scan rate of $0.5 \mathrm{mV} \mathrm{s}^{-1}$.

To achieve real battery cycling and nanoscale visualization in an identical configuration, a TEM grid loaded with $\mathrm{NiO}$ nanosheets was pressed against the composite electrode (Fig. 1). The TEM grid was carefully removed and rinsed with diethyl carbonate after the designated electrochemical cycling and allowed to dry for an extended period of time in the glove box. Composite electrodes on copper foils and TEM grids were transferred under Ar protection for ensemble-averaged
} and spatially resolved measurements, respectively.
Synchrotron XAS. High-throughput XAS measurements were performed on the 31-pole wiggler beamline 10-1 at Stanford Synchrotron Radiation Lightsource using a ring current of $350 \mathrm{~mA}$ and a $1,000 \mathrm{~mm}^{-1}$ spherical grating monochromator with $20 \mu \mathrm{m}$ entrance and exit slits, providing $\sim 10^{11} \mathrm{ph} \mathrm{s}^{-1}$ at $0.2 \mathrm{eV}$ resolution in a $1 \mathrm{~mm}^{2}$ beam spot. During the measurements, all samples were attached to an aluminium sample holder, and the surface was connected to the isolated holder using conductive carbon. Data were acquired in a single load at room temperature and under ultrahigh vacuum $\left(10^{-9}\right.$ torr). For TEY, we collected the sample drain current, and a silicon diode (IRD AXUV-100) was used to collect the fluorescence yield positioned near the sample surface. AEY was collected with a Cylindrical Mirror Analyser using a pass energy of $200 \mathrm{eV}$ and a kinetic energy window of $2 \mathrm{eV}$ near the main Auger for oxygen and nickel, respectively. Contributions from visible light were carefully minimized before the acquisition, and all spectra were normalized by the current from freshly evaporated gold on a fine grid positioned upstream of the main chamber. Samples were prepared by loading battery electrodes onto a conductive carbon tape, which was mounted on an aluminium holder attached to the main chamber manipulator.

Electron microscopy and spectroscopy. 200 and $300 \mathrm{keV}$ field-emission S/TEMs were used for ADF-STEM tomographic imaging. EELS data sets were acquired using a Gatan Tridiem spectrometer. Tilt series images were recorded from $-70^{\circ}$ to $+70^{\circ}$ with $1^{\circ}$ intervals. Tomograms were reconstructed using the $\mathrm{e}^{-}$Tomo software suite written by Robert Hovden et al. (Cornell Muller group) and visualized by Avizo 6.3.

\section{References}

1. Anderson, V. J. \& Lekkerkerker, H. N. W. Insights into phase transition kinetics from colloid science. Nature 416, 811-815 (2002).

2. Armstrong, A. R., Lyness, C., Panchmatia, P. M., Islam, M. S. \& Bruce, P. G. The lithium intercalation process in the low-voltage lithium battery anode $\mathrm{Li}_{1+\mathrm{x}} \mathrm{V}_{1-\mathrm{x}} \mathrm{O}_{2}$. Nat. Mater. 10, 223-229 (2011).

3. Malik, R., Zhou, F. \& Ceder, G. Kinetics of non-equilibrium lithium incorporation in $\mathrm{LiFePO}_{4}$. Nat. Mater. 10, 587-590 (2011).

4. Wang, D. et al. Structurally ordered intermetallic platinum-cobalt core-shell nanoparticles with enhanced activity and stability as oxygen reduction electrocatalysts. Nat. Mater. 12, 81-87 (2013).

5. Wang, D. et al. Tuning oxygen reduction reaction activity via controllable dealloying: a model study of ordered $\mathrm{Cu}_{3} \mathrm{Pt} / \mathrm{C}$ intermetallic nanocatalysts. Nano Lett. 12, 5230-5238 (2012).

6. Tang, M., Carter, W. C. \& Chiang, Y.-M. Electrochemically driven phase transitions in insertion electrodes for lithium-ion batteries: examples in lithium metal phosphate olivines. Annu. Rev. Mater. Res. 40, 501-529 (2010).

7. Wang, F. et al. Conversion reaction mechanisms in lithium ion batteries: study of the binary metal fluoride electrodes. J. Am. Chem. Soc. 133, 18828-18836 (2011).

8. Huang, J. Y. et al. In situ observation of the electrochemical lithiation of a single $\mathrm{SnO}_{2}$ nanowire electrode. Science 330, 1515-1520 (2010).

9. Levi, M. D. et al. Collective phase transition dynamics in microarray composite $\mathrm{Li}_{\mathrm{x}} \mathrm{FePO}_{4}$ electrodes tracked by in situ electrochemical quartz crystal admittance. J. Phys. Chem. C 117, 15505-15514 (2013).

10. Chueh, W. C. et al. Intercalation pathway in many-particle $\mathrm{LiFePO}_{4}$ electrode revealed by nanoscale state-of-charge mapping. Nano Lett. 13, 866-872 (2013)

11. Wang, F. et al. Tracking lithium transport and electrochemical reactions in nanoparticles. Nat. Commun. 3, 1201 (2012).

12. Delmas, C., Maccario, M., Croguennec, L., Le Cras, F. \& Weill, F. Lithium deintercalation in $\mathrm{LiFePO}_{4}$ nanoparticles via a domino-cascade model. Nat. Mater. 7, 665-671 (2008).

13. Sun, Y. et al. Direct atomic-scale confirmation of three-phase storage mechanism in $\mathrm{Li}_{4} \mathrm{Ti}_{5} \mathrm{O}_{12}$ anodes for room-temperature sodium-ion batteries. Nat. Commun. 4, 1870 (2013).

14. Li, C., Gu, L., Tsukimoto, S., van Aken, P. A. \& Maier, J. Low-temperature ionic-liquid-based synthesis of nanostructured iron-based fluoride cathodes for lithium batteries. Adv. Mater. 22, 3650-3654 (2010).

15. Oumellal, Y., Rougier, A., Nazri, G. A., Tarascon, J.-M. \& Aymard, L. Metal hydrides for lithium-ion batteries. Nat. Mater. 7, 916-921 (2008).

16. Li, H., Liu, X., Zhai, T., Li, D. \& Zhou, H. $\mathrm{Li}_{3} \mathrm{VO}_{4}$ : a promising insertion anode material for lithium-ion batteries. Adv. Energy Mater. 3, 428-432 (2013).

17. Lee, S.-H. et al. Reversible lithium-ion insertion in molybdenum oxide nanoparticles. Adv. Mater. 20, 3627-3632 (2008).

18. Cabana, J., Monconduit, L., Larcher, D. \& Palacín, M. R. Beyond intercalationbased Li-ion batteries: the state of the art and challenges of electrode materials reacting through conversion reactions. Adv. Mater. 22, E170-E192 (2010).

19. Reddy, M. V., Subba Rao, G. V. \& Chowdari, B. V. R. Metal oxides and oxysalts as anode materials for li ion batteries. Chem. Rev. 113, 5364-5457 (2013).

20. Poizot, P., Laruelle, S., Grugeon, S., Dupont, L. \& Tarascon, J. M. Nano-sized transition-metal oxides as negative-electrode materials for lithium-ion batteries. Nature 407, 496-499 (2000). 
21. Ban, C. et al. Nanostructured $\mathrm{Fe}_{3} \mathrm{O}_{4} / \mathrm{SWNT}$ electrode: Binder-free and highrate li-ion anode. Adv. Mater. 22, E145-E149 (2010).

22. Balaya, P., Li, H., Kienle, L. \& Maier, J. Fully Reversible Homogeneous and Heterogeneous Li Storage in $\mathrm{RuO}_{2}$ with High Capacity. Adv. Funct. Mater. 13, 621-625 (2003).

23. Gao, J., Lowe, M. A. \& Abruña, H. D. Spongelike nanosized $\mathrm{Mn}_{3} \mathrm{O}_{4}$ as a high-capacity anode material for rechargeable lithium batteries. Chem. Mater. 23, 3223-3227 (2011)

24. Dupont, L. et al. Mesoporous $\mathrm{Cr}_{2} \mathrm{O}_{3}$ as negative electrode in lithium batteries: TEM study of the texture effect on the polymeric layer formation. J. Power Sources 175, 502-509 (2008).

25. Zhou, G. et al. Oxygen bridges between $\mathrm{NiO}$ nanosheets and graphene for improvement of lithium storage. ACS Nano 6, 3214-3223 (2012).

26. Varghese, B. et al. Fabrication of $\mathrm{NiO}$ nanowall electrodes for high performance lithium ion battery. Chem. Mater. 20, 3360-3367 (2008).

27. Liu, L. et al. Nanosheet-based $\mathrm{NiO}$ microspheres: controlled solvothermal synthesis and lithium storage performances. J. Phys. Chem. C 114, 251-255 (2010).

28. Etacheri, V., Marom, R., Elazari, R., Salitra, G. \& Aurbach, D. Challenges in the development of advanced Li-ion batteries: a review. Energy Environ. Sci. 4, 3243-3262 (2011).

29. Harris, S. J. \& Lu, P. Effects of inhomogeneities-nanoscale to mesoscale-on the durability of li-ion batteries. J. Phys. Chem. C 117, 6481-6492 (2013).

30. Borkiewicz, O. J., Chapman, K. W. \& Chupas, P. J. Mapping spatially inhomogeneous electrochemical reactions in battery electrodes using high energy X-rays. Phys. Chem. Chem. Phys. 15, 8466-8469 (2013).

31. Yu, Y. et al. Three-dimensional tracking and visualization of hundreds of Pt-Co fuel cell nanocatalysts during electrochemical aging. Nano Lett. 12, 4417-4423 (2012).

32. $\mathrm{Hu}$, J. et al. Preparation and surface activity of single-crystalline $\mathrm{NiO}(111)$ nanosheets with hexagonal holes: a semiconductor nanospanner. Adv. Mater. 20, 267-271 (2008).

33. Lin, F. et al. Origin of electrochromism in high-performing nanocomposite nickel oxide. ACS Appl. Mater. Interfaces 5, 3643-3649 (2013).

34. Lin, F. et al. Hole doping in Al-containing nickel oxide materials to improve electrochromic performance. ACS Appl. Mater. Interfaces 5, 301-309 (2013).

35. Soriano, L. et al. The electronic structure of mesoscopic NiO particles. Chem. Phys. Lett. 208, 460-464 (1993).

36. Zhukovskii, Y. F., Balaya, P., Kotomin, E. A. \& Maier, J. Evidence for interfacial-storage anomaly in nanocomposites for lithium batteries from firstprinciples simulations. Phys. Rev. Lett. 96, 058302 (2006).

37. Fransson, L., Eriksson, T., Edström, K., Gustafsson, T. \& Thomas, J. Influence of carbon black and binder on Li-ion batteries. J. Power Sources 101, 1-9 (2001).

38. Sahay, R. et al. High aspect ratio electrospun cuo nanofibers as anode material for lithium-ion batteries with superior cycleability. J. Phys. Chem. C 116, 18087-18092 (2012)

39. Hu, Y.-Y. et al. Origin of additional capacities in metal oxide lithium-ion battery electrodes. Nat. Mater. 12, 1130-1136 (2013).

40. Su, D., Ford, M. \& Wang, G. Mesoporous NiO crystals with dominantly exposed $\{110\}$ reactive facets for ultrafast lithium storage. Sci. Rep. 2, 924 (2012).

41. Qiao, R., Chuang, Y.-D., Yan, S. \& Yang, W. Soft X-ray irradiation effects of $\mathrm{Li}_{2} \mathrm{O}_{2}, \mathrm{Li}_{2} \mathrm{CO}_{3}$ and $\mathrm{Li}_{2} \mathrm{O}$ revealed by absorption spectroscopy. PLoS One 7, e49182 (2012)

42. Garvie, L. A. J. \& Graven, A. J. Use of electron-energy loss near-edge fine structure in the study of minerals. Am. Mineral. 79, 411-425 (1994).

43. Wang, F. et al. Chemical distribution and bonding of lithium in intercalated graphite: identification with optimized electron energy loss spectroscopy. ACS Nano 5, 1190-1197 (2011).

44. Dollé, M., Grugeon, S., Beaudoin, B., Dupont, L. \& Tarascon, J.-M. In situ TEM study of the interface carbon/electrolyte. J. Power Sources 97, 104-106 (2001).

45. Xu, K. Nonaqueous liquid electrolytes for lithium-based rechargeable batteries. Chem. Rev. 104, 4303-4418 (2004).
46. Lu, P. \& Harris, S. J. Lithium transport within the solid electrolyte interphase. Electrochem. Commun. 13, 1035-1037 (2011).

47. Shi, S. et al. Direct calculation of Li-ion transport in the solid electrolyte interphase. J. Am. Chem. Soc. 134, 15476-15487 (2012).

48. Ebner, M., Marone, F., Stampanoni, M. \& Wood, V. Visualization and quantification of electrochemical and mechanical degradation in li ion batteries. Science 342, 716-720 (2013).

49. Shyam, B. et al. Structural and mechanistic revelations on an iron conversion reaction from pair distribution function analysis. Angew. Chem. Int. Ed. 51, 4852-4855 (2012).

50. Bruce, P. G., Scrosati, B. \& Tarascon, J.-M. Nanomaterials for rechargeable lithium batteries. Angew. Chem. Int. Ed. 47, 2930-2946 (2008).

51. Liu, H., Wang, G., Liu, J., Qiao, S. \& Ahn, H. Highly ordered mesoporous NiO anode material for lithium ion batteries with an excellent electrochemical performance. J. Mater. Chem. 21, 3046-3052 (2011).

52. Chen, Q. \& Sieradzki, K. Spontaneous evolution of bicontinuous nanostructures in dealloyed Li-based systems. Nat. Mater. 12, 1102-1106 (2013).

53. Meirer, F. et al. Three-dimensional imaging of chemical phase transformations at the nanoscale with full-field transmission X-ray microscopy. J. Synchrotron Radiat. 18, 773-781 (2011).

54. Porter, D. A. \& Easterling, K. E. Phase Transformations in Metals and Alloys 514 (Nelson Thornes, 1992).

55. Gregorczyk, K. E., Liu, Y., Sullivan, J. P. \& Rubloff, G. W. In situ transmission electron microscopy study of electrochemical lithiation and delithiation cycling of the conversion anode $\mathrm{RuO}_{2}$. ACS Nano 7, 6354-6360 (2013).

\section{Acknowledgements}

The synchrotron X-ray portions of this research were carried out at the Stanford Synchrotron Radiation Lightsource, a Directorate of SLAC National Accelerator Laboratory and an Office of Science User Facility operated for the US Department of Energy Office of Science by Stanford University. S/TEM, tomography and EELS experiments were performed at the Center for Functional Nanomaterials, Brookhaven National Laboratory, which is supported by the US Department of Energy, Office of Basic Energy Sciences under Contract No. DE-AC02-98CH10886, and at National Center for Electron Microscopy (NCEM) of the Lawrence Berkeley National Laboratory (LBNL), which is supported by the US Department of Energy (DOE) under Contract No. DE-AC02-05CH11231. R.M.R. thanks the Colorado School of Mines for financial support. F.L. and H.L.X. would like to acknowledge Dr Marca Doeff (LBNL) for her generosity in providing equipments for some portion of the study, and Hao Zou (Hella) for the coin cell artwork. H.L.X. also would like to thank Robert Hovden and Julia Mundy for their input.

\section{Author contributions}

F.L. and H.L.X. conceived and designed the experiments. F.L. performed synthesis, carried out electrochemical tests and prepared samples for XAS and electron microscopy. F.L., D.N. and T.-C.W. performed synchrotron X-ray absorption experiments. H.L.X. performed S/TEM, EELS and tomography experiments. Y.Z., C.B. and R.M.R. participated in data analysis. F.L. wrote the manuscript and prepared the figures with assistance from all authors. H.L.X. led the research project. All authors participated in discussions and know implications of the work.

\section{Additional information}

Supplementary Information accompanies this paper at http://www.nature.com/ naturecommunications

Competing financial interests: The authors declare no competing financial interests.

Reprints and permission information is available online at http://npg.nature.com/ reprintsandpermissions/

How to cite this article: Lin, F. et al. Phase evolution for conversion reaction electrodes in lithium-ion batteries. Nat. Commun. 5:3358 doi: 10.1038/ncomms4358 (2014). 